\title{
Decrease in skeletal muscle index one year after radical cystectomy as a prognostic indicator in patients with urothelial bladder cancer
}

\author{
Yun-Sok Ha ${ }^{1}$, Sang Won Kim ${ }^{1}$, Tae Gyun Kwon ${ }^{1}$, Sung Kwang Chung ${ }^{1}$, Eun Sang Yoo ${ }^{1}$ \\ ${ }^{1}$ Department of Urology, School of Medicine, Kyungpook National University, Daegu, Republic of Korea
}

\section{ABSTRACT}

Purpose: The present study aimed to determine whether sarcopenia after radical cystectomy $(\mathrm{RC})$ could predict overall survival (OS) in patients with urothelial bladder cancer (UBC).

Materials and Methods: The lumbar skeletal muscle index (SMI) of 80 patients was measured before and 1 year after RC. The prognostic significance of sarcopenia and SMI decrease after RC were evaluated using Kaplan-Meier analysis and a multivariable Cox regression model.

Results: Of 80 patients, 26 (32.5\%) experienced sarcopenia before RC, whereas 40 (50.0\%) experienced sarcopenia after RC. The median SMI change was $-2.2 \mathrm{~cm} 2 / \mathrm{m} 2$. Patients with sarcopenia after RC had a higher pathological T stage and tumor grade than patients without sarcopenia. Furthermore, the overall mortality rate was significantly higher in patients with sarcopenia than in those without sarcopenia 1 year after RC. The median follow-up time was 46.2 months, during which 22 patients died. Kaplan-Meier estimates showed a significant difference in OS rates based on sarcopenia $(\mathrm{P}=0.012)$ and SMI decrease $(\mathrm{P}=0.025)$. Multivariable Cox regression analysis showed that SMI decrease $(\geq 2.2 \mathrm{~cm} 2 / \mathrm{m} 2)$ was an independent predictor of OS (hazard ratio: 2.68, confidence interval: 1.007-7.719, $\mathrm{P}=0.048$ ).

Conclusions: The decrease in SMI after surgery might be a negative prognostic factor for OS in patients who underwent RC to treat UBC.

\section{ARTICLE INFO}

Yun-Sok Ha

https://orcid.org/0000-0003-3732-9814

\section{Keywords:}

Urinary Bladder Neoplasms;

Sarcopenia; Survival

Int Braz J Urol. 2019; 45: 686-94

Submitted for publication:

July 16,2018

Accepted after revision:

December 14, 2018

Published as Ahead of Print:

March 20, 2019

\section{INTRODUCTION}

Bladder cancer (BC) is one of the most common urinary tract malignancies worldwide (1-3). It is generally treated using transurethral resection of bladder tumor (TUR-BT) or radical cystectomy (RC), and systemic cisplastin-based chemotherapy is performed in cases of advanced or metastatic BC. However, because BC is a highly malignant tumor with a variable and unpredic- table biologic potential, the survival forecast for patients remains poor $(4,5)$ The prognosis of $\mathrm{BC}$ is poor in elderly people and in those with serious comorbidities and poor performance status (6).

$\mathrm{RC}$ is the customary treatment for patients with muscle-invasive $\mathrm{BC}$ (MIBC), and it is also commonly used to treat selected patients with high-risk, non-muscle-invasive BC (NMI$\mathrm{BC}$ ). A recent study reported that complications following RC are strongly associated with patient- 
-related factors, such as age, performance status, and comorbidities (7). Moreover, numerous studies have demonstrated that frailty is associated with impaired mobility, disability, poor endurance, and prolonged hospitalization $(8,9)$. In particular, sarcopenia-skeletal muscle wasting- is a crucial physiological alteration underlying frailty that can emerge as a result of aging and malignant disease (10), and it has been identified as a prognostic factor for various cancers (11). In patients with $\mathrm{BC}$ who have undergone RC, sarcopenia is associated with poor survival (12). However, changes in the skeletal muscle index (SMI) after RC have not been established as a prognostic tool. The aim of the present study was to evaluate changes in the SMI 1 year after RC as a predictor of overall survival (OS) in patients with urothelial bladder cancer (UBC).

\section{MATERIALS AND METHODS}

\section{Ethics statement}

The ethics committee of Kyungpook National University Hospital reviewed and approved the current study protocol (approval number: KNUMC 2016-05-021). The study was performed in accordance with the ethical standards laid down in the 1964 Declaration of Helsinki and its later amendments. This was a retrospective study performed after approval from the institutional review board, who stated that consent was not required.

\section{Patients}

The present study included 80 patients with non-metastatic UBC who had undergone RC (31 robot-assisted RCs and 49 open RCs) between August 2008 and May 2013 and who had serial axial computed tomography (CT) images showing sarcopenia both before and 1 year after the RC. Before RC, all patients underwent TUR-BT. Following histopathological examination and imaging studies, RC was performed. The indications for RC were as follows: MIBC without evidence of distant metastasis (clinical stage: T2-T4, Nx, M0), recurrent multifocal NMIBC refractory to repeated transurethral resection, and Bacille Calmette-Guerin (BCG)-resistant carcinoma in situ. The exclusion criteria were as follows: previous pelvic radiation, clinical stage M1, and prior combination surgery.
Open RC was performed through a midline incision in the typical manner (13). Robot-assisted RC was performed using the same surgical procedure as reported by Bak et al. (1). Standard pelvic lymphadenectomy (both obturator and external iliac nodes) was performed in all patients, except for 1 patient undergoing robot-assisted RC and 6 undergoing open RC because of severe adhesions. The clinical T stage was based on the guidelines of the 2010 American Joint Committee on Cancer TNM staging system for BC (14). Histological grades were determined according to the 2004 World Health Organization (WHO) classification system (15). Patients with cT3, cT4, and node-positive disease (based on the analysis of CT images), but with good performance status, received at least 3 cycles of cisplatin-based neoadjuvant chemotherapy. Each patient was followed up and managed according to standard practice (16).

\section{Image analysis}

Patients underwent abdominal CT for initial cancer staging and routine diagnostic purposes. For each patient, a set of CT scans just before and a mean of 1 year after RC was selected. Quantitative assessment of muscle areas was performed using commercially available software (Terarecon 4.4.7, San Mateo, CA, USA) by a subspecialty-trained urogenital radiologist. The radiologist selected the single cross-sectional areas at the level of the third lumbar vertebrae (L3) in which both transverse processes could be fully seen. The cross-sectional areas $\left(\mathrm{cm}^{2}\right)$ of all skeletal muscles at L3 were computed automatically by summing the appropriate pixels within the CT Hounsfield unit (HU) range of -29 HU to $150 \mathrm{HU}$ (17). After applying a predefined HU threshold set for each slice, boundaries between the different tissues were corrected manually when necessary.

\section{Definition of sarcopenia}

Muscle area was normalized for the square of patient height in meters $\left(\mathrm{m}^{2}\right)$ and reported as the lumbar SMI index $\left(\mathrm{cm}^{2} / \mathrm{m}^{2}\right)(18,19)$. In Figure- 1 shows the CT scans and SMI values of an 82-year-old man before and at a mean of 1 year after RC. Sarcopenia was defined as a lumbar SMI of $<43 \mathrm{~cm}^{2} / \mathrm{m}^{2}$ for men with a body mass index 
Figure 1 - Representative CT scans with decreased skeletal muscle index (SMI) in an 82-year-old man before (A) and at a mean of one year (B) after radical cystectomy.

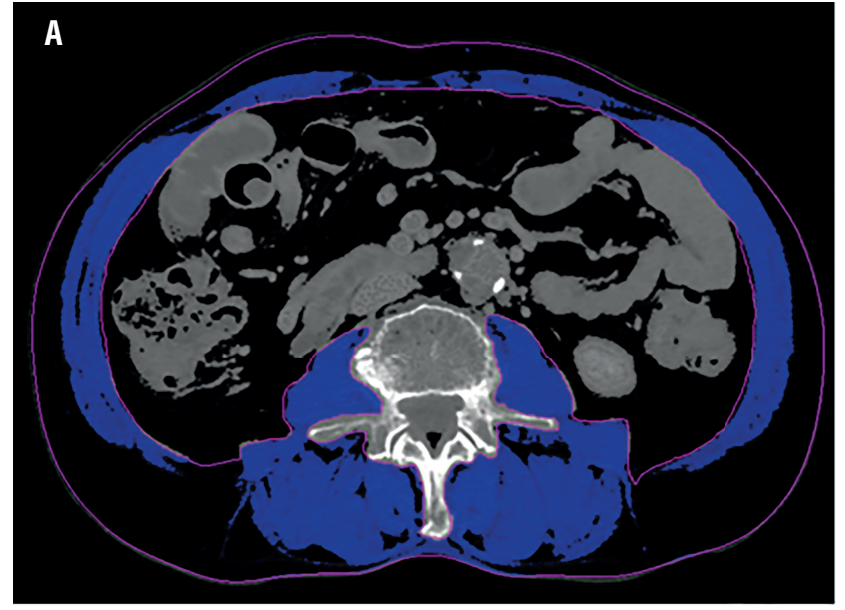

SMI $47 \mathrm{~cm}^{2} / \mathrm{m}^{2}$

(BMI) of $<25 \mathrm{~kg} / \mathrm{m}^{2}$, as a lumbar SMI of $<53 \mathrm{~cm}^{2} /$ $\mathrm{m}^{2}$ for those with a BMI of $\geq 25 \mathrm{~kg} / \mathrm{m}^{2}$, and as an SMI of $<41 \mathrm{~cm}^{2} / \mathrm{m}^{2}$ for women, as recommended by Martin et al. (20).

\section{Statistical analysis}

Patients were divided into two groups of 40 based on their sarcopenic status one year after RC: non-sarcopenic patients and sarcopenic patients. Differences between the groups were evaluated using the chi-square test for categorical variables and Student's t-test for continuous variables. Multivariable Cox proportional hazards models were used to test the associations between the variables and OS, with hazard ratios (HRs) and 95\% confidence intervals (CIs) calculated for each factor. OS was measured from the date of diagnosis to death or final follow-up. All statistical analyses were performed using the Statistical Package for the Social Sciences, version 18.0 (SPSS Inc., Chicago, IL, USA), and P-values $<0.05$ were considered statistically significant.

\section{RESULTS}

Of the 80 patients, 26 (32.5\%) were sarcopenic before RC, whereas 40 (50.0\%) were sarcopenic after RC. The median change in SMI was -2.2

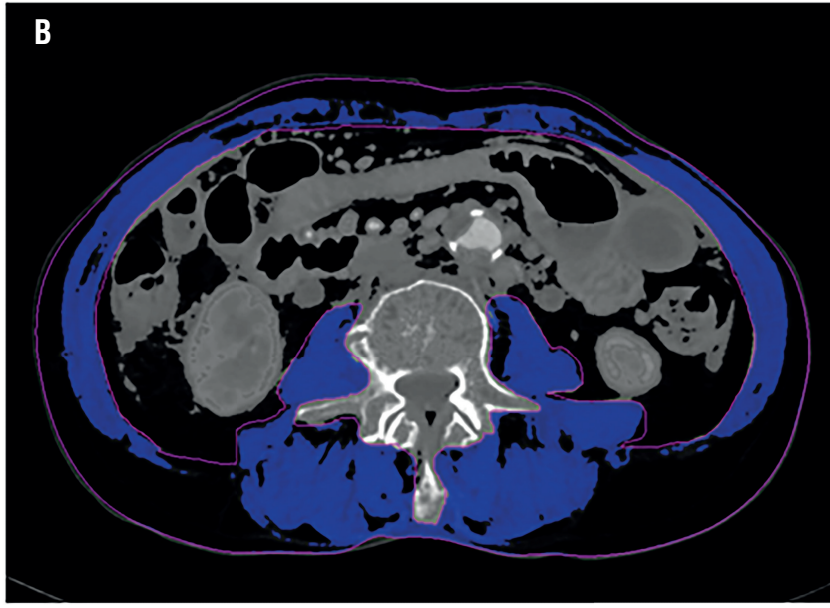

SMI $38.1 \mathrm{~cm}^{2} / \mathrm{m}^{2}$

$\mathrm{cm}^{2} / \mathrm{m}^{2}$. In Table- 1 presents patient demographics and preoperative characteristics according to SMI 1 year after RC. Age, sex, and BMI were not significantly associated with sarcopenia 1 year after RC $(P>0.05)$. The mean preoperative SMI was 50.51 $\mathrm{cm}^{2} / \mathrm{m}^{2}$ in non-sarcopenic patients, significantly higher than that in sarcopenic patients (43.76 $\left.\mathrm{cm}^{2} / \mathrm{m}^{2} ; P<0.001\right)$. In the cohort of patients with sarcopenia after RC, $47.5 \%$ had been classified as sarcopenic preoperatively, while only 7 (17.5\%) patients with preoperative sarcopenia were in the non-sarcopenic group after surgery $(P=0.004)$. Higher clinical stage $(\geq \mathrm{T} 2)$ at latest TUR-BT was more prevalent in sarcopenic patients than in those without sarcopenia (65.0\% vs. 37.5\%; $P=0.014)$. Sarcopenia was not significantly associated with ASA classification, presence of carcinoma in situ, BCG instillation history, and neoadjuvant chemotherapy.

In Table- 2 shows the relationship between sarcopenia after RC and clinicopathological features. Evaluation revealed that patients with sarcopenia had higher tumor stage and grade than those without sarcopenia 1 year after RC. The mean changes in SMI were $-3.80 \mathrm{~cm}^{2} / \mathrm{m}^{2}$ in sarcopenic patients and $-1.12 \mathrm{~cm}^{2} / \mathrm{m}^{2}$ in non-sarcopenic patients $(P=0.001)$. Notably, patients with sarcopenia had significantly higher all-cause mortality rates than those without sarcopenia $(P=0.012)$. Metas- 
Table 1 - Patient demographics and preoperative characteristics according to skeletal muscle index one year after radical cystectomy.

\begin{tabular}{|c|c|c|c|}
\hline Parameters & Non-sarcopenic patients, $(n=40)$ & Sarcopenic patients, $(n=40)$ & $P$-value \\
\hline Age, years & & & 0.262 \\
\hline$<70$ & $24(60.0)$ & $19(47.5)$ & \\
\hline$\geq 70$ & $16(40.0)$ & $21(52.5)$ & \\
\hline Sex & & & 0.363 \\
\hline Male & $35(87.5)$ & $32(80.0)$ & \\
\hline Female & $5(12.5)$ & $8(20.0)$ & \\
\hline $\mathrm{BMI}\left(\mathrm{kg} / \mathrm{m}^{2}\right)$ & $23.41 \pm 3.41$ & $22.39 \pm 2.71$ & 0.142 \\
\hline Preoperative SMI $\left(\mathrm{cm}^{2} / \mathrm{m}^{2}\right)$ & $50.51 \pm 8.41$ & $43.76 \pm 6.40$ & $<0.001$ \\
\hline Preoperative sarcopenia & & & 0.004 \\
\hline No & $33(82.5)$ & $21(52.5)$ & \\
\hline Yes & $7(17.5)$ & $19(47.5)$ & \\
\hline ASA classification & & & 0.499 \\
\hline 1 & $6(15.0)$ & $4(10.0)$ & \\
\hline$\geq 2$ & $34(85.0)$ & $36(90.0)$ & \\
\hline Clinical stage at latest TUR-BT & & & 0.014 \\
\hline$\leq \mathrm{T} 1$ & $25(62.5)$ & $14(35.0)$ & \\
\hline$\geq \mathrm{T} 2$ & $15(37.5)$ & $26(65.0)$ & \\
\hline Presence of CIS at latest TUR-BT & & & 0.712 \\
\hline No & $35(87.5)$ & $37(92.5)$ & \\
\hline Yes & $5(12.5)$ & $3(7.5)$ & \\
\hline BCG instillation history & & & 0.712 \\
\hline No & $37(92.5)$ & $35(87.5)$ & \\
\hline Yes & $3(7.5)$ & $5(12.5)$ & \\
\hline Neoadjuvant chemotherapy & & & 0.762 \\
\hline No & $34(85.0)$ & $33(82.5)$ & \\
\hline Yes & $6(15.0)$ & $7(17.5)$ & \\
\hline
\end{tabular}

$\mathbf{A S A}=$ American Society of Anesthesiologists; $\mathbf{B C G}=$ Bacille Calmette-Guerin; $\mathbf{B M I}=$ body mass index; $\mathbf{C I S}=$ Carcinoma in situ; $\mathbf{S M I}$ = Skeletal muscle index; $\mathbf{T U R}-\mathbf{B T}$ $=$ Transurethral tumor resection of bladder tumor

tasis rate did not differ significantly between the 2 groups $(P=0.104)$.

Twenty-two patients died during the median follow-up of 46.2 months. Kaplan-Meier estimates showed a significant difference in OS $(P=0.012)$ and SMI decrease $(P=0.025)$ between the 2 groups (Figure-2). In Table-3, we assessed the relationship between various measured pa- rameters and OS. Although sarcopenia 1 year after RC was significantly associated with OS in univariable analysis, there was no statistical association between sarcopenia itself and OS in multivariable Cox analysis. As indicated by the multivariable analysis, the probability of OS increased greatly as SMI decreased. When other factors were adjusted for, pathological $\mathrm{T}$ stage 
Table 2 - Comparison of clinicopathological variables according to skeletal muscle index one year after radical cystectomy.

\begin{tabular}{|c|c|c|c|}
\hline Parameters & $\begin{array}{l}\text { Non-sarcopenic patients, } \\
\qquad(n=40)\end{array}$ & $\begin{array}{l}\text { Sarcopenic patients, } \\
\qquad(n=40)\end{array}$ & P-value \\
\hline Pathological stage & & & 0.028 \\
\hline T0, Tis, Ta & $6(15.0)$ & $2(5.0)$ & \\
\hline $\mathrm{T} 1$ & $15(37.5)$ & $10(25.0)$ & \\
\hline T2 & $10(25.0)$ & $9(22.5)$ & \\
\hline T3 & $4(10.0)$ & $13(32.5)$ & \\
\hline $\mathrm{T} 4$ & $5(12.5)$ & $6(15.0)$ & \\
\hline Histological grade & & & 0.029 \\
\hline Low & $8(20.0)$ & $1(2.5)$ & \\
\hline High & $32(80.0)$ & $39(97.5)$ & \\
\hline Lymph node involvement & & & 0.617 \\
\hline No & $28(70.0)$ & $30(75.0)$ & \\
\hline Yes & $12(30.0)$ & $10(25.0)$ & \\
\hline Lymphovascular invasion & & & 0.712 \\
\hline No & $37(92.5)$ & $35(87.5)$ & \\
\hline Yes & $3(7.5)$ & $5(12.5)$ & \\
\hline SMI changes 1 year after $\mathrm{RC}\left(\mathrm{cm}^{2} / \mathrm{m}^{2}\right)$ & $-1.12 \pm 3.14$ & $-3.80 \pm 3.59$ & 0.001 \\
\hline $\begin{array}{l}\text { Median follow-up period (months, } \\
\text { range) }\end{array}$ & $48.1(14.4-105.1)$ & $43.5(12.5-93.0)$ & 0.060 \\
\hline Metastasis & & & 0.104 \\
\hline No & $29(72.5)$ & $22(55.0)$ & \\
\hline Yes & $11(27.5)$ & $18(45.0)$ & \\
\hline Overall death & & & 0.012 \\
\hline No & $34(85.0)$ & $24(60.0)$ & \\
\hline Yes & $6(15.0)$ & $16(40.0)$ & \\
\hline
\end{tabular}

and an SMI decrease of $\geq 2.2 \mathrm{~cm}^{2} / \mathrm{m}^{2}$ (HR: 2.689, 95\% CI: $1.007-7.719, P=0.048$ ) were found to be independent predictors of OS.

\section{DISCUSSION}

Muscle loss is expected in the elderly and is a rising concern in patients with cancer. Sarcopenia is characterized by decrease in protein synthesis and an increase in protein degradation (21). Hence, the condition displays many similar characteristics and can be a broad and integrated sign of cancer cachexia. Several re- cent studies have revealed definite connections between sarcopenia and mortality after RC to treat UBC $(12,22)$. To our knowledge, our current report was the first to indicate that changes in SMI after RC are associated with OS in patients with UBC. In particular, we observed greater all-cause mortality among patients who were sarcopenic after RC to treat UBC (40.0\% vs. $15.0 \%$ in non-sarcopenic patients; $P=0.012$ ). The median OS was 43.5 months among patients with sarcopenia versus 48.1 months among those with normal SMI after RC. All-cause mortality was more prevalent in the sarcopenia group 
Figure 2 - Kaplan-Meier curve depicting overall survival according to postoperative sarcopenia $(A)$ and change in skeletal muscle index (SMI) (B).

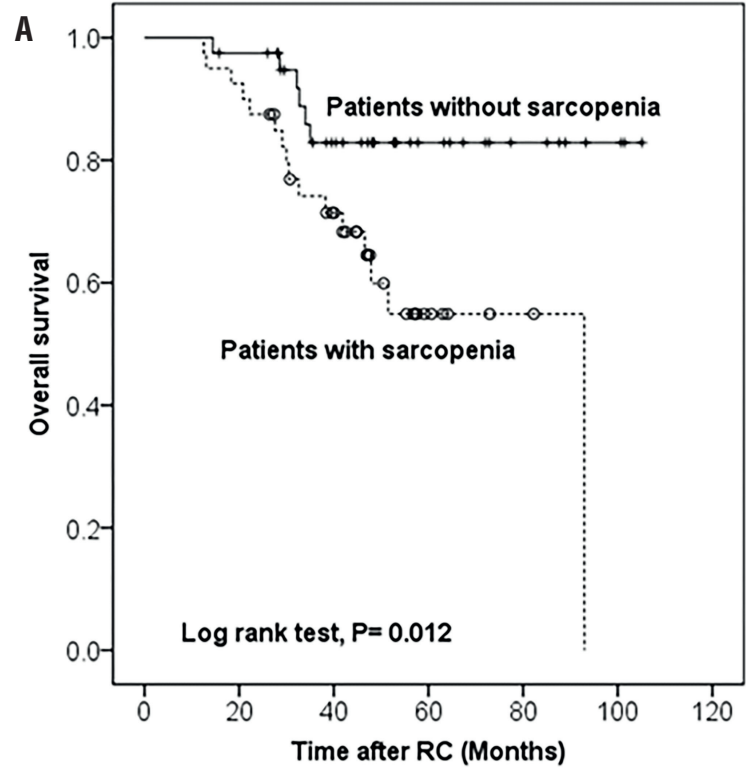

than in the cohort without sarcopenia, according to Kaplan-Meier analysis (log-rank test: $P=0.012$; Figure-2A). Likewise, Kaplan-Meier analysis also revealed that patients with larger SMI changes $\left(\geq 2.2 \mathrm{~cm}^{2} / \mathrm{m}^{2}\right)$ had a worse OS rate than those with smaller changes $\left(<2.2 \mathrm{~cm}^{2} / \mathrm{m}^{2} ; \log \right.$ rank test; $P=0.0025$; Figure-2B). In our multivariable analysis, larger SMI decreases $\left(\geq 2.2 \mathrm{~cm}^{2} / \mathrm{m}^{2}\right)$ were associated with the risk of all-cause mortality (HR: 2.689, 95\% CI: 1.007-7.719, $P=0.048$ ).

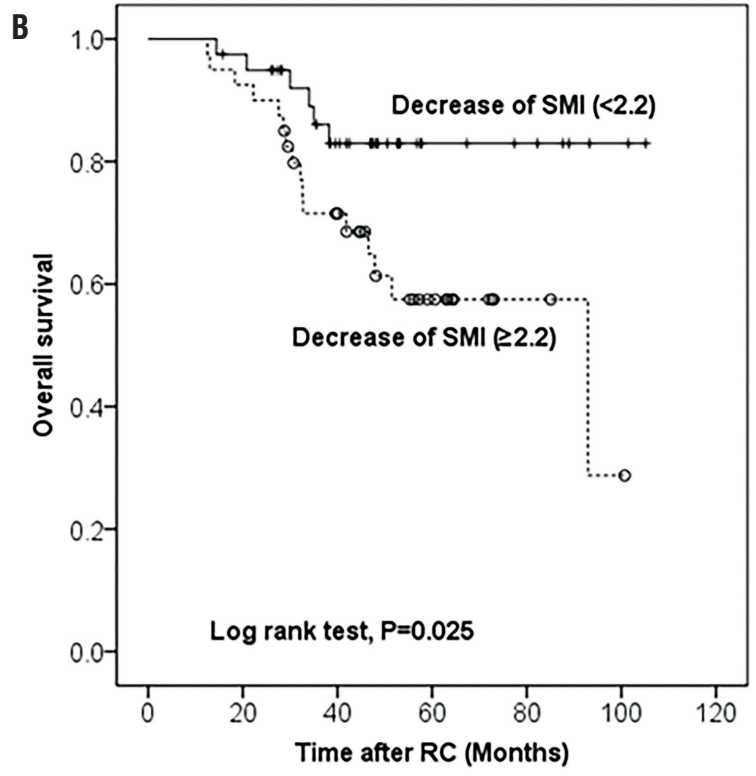

Taken together with our results, sarcopenia and decreased SMI after RC are clinically useful and highly objective predictors of OS in patients with UBC who have undergone RC. Although sarcopenia one year after RC was significantly associated with OS in univariable analysis, there was no statistical association between sarcopenia itself and OS in multivariable Cox analysis. This suggested that the change in SMI was more useful for prediction of the OS after RC.

Table 3 - Multivariable Cox regression analysis of factors predicting overall death in patients with bladder cancer after radical cystectomy.

\begin{tabular}{lllllllll}
\hline Parameters & HR & $95 \% \mathrm{Cl}$ & & P-value & HR & $95 \% \mathrm{Cl}$ & & $P$-value \\
& & & & & & & & \\
\hline Age & 1.002 & 0.955 & 1.050 & 0.943 & 1.017 & 0.974 & 1.063 & 0.434 \\
Gender & 0.359 & 0.078 & 1.660 & 0.190 & 0.615 & 0.137 & 2.768 & 0.527 \\
Pathological T stage & 1.835 & 1.191 & 2.827 & 0.006 & 1.664 & 1.062 & 2.607 & 0.026 \\
Lymph node involvement & 1.329 & 0.515 & 3.424 & 0.556 & 1.719 & 0.675 & 4.383 & 0.256 \\
Grade & 1.315 & 0.133 & 12.977 & 0.815 & 1.381 & 0.149 & 12.815 & 0.776 \\
Sarcopenia 1 year after RC (No vs. Yes) & 1.714 & 0.645 & 4.558 & 0.280 & & & & \\
SMI decrease (<2.2 vs. $\left.\geq 2.2 \mathrm{~cm}^{2} / \mathrm{m}^{2}\right)$ & & & & & 2.689 & 1.007 & 7.719 & 0.048 \\
\hline
\end{tabular}

$\mathbf{C I}=$ confidence interval; $\mathbf{H R}=$ hazard ratio; $\mathbf{S M I}=$ skeletal muscle index 
In this regard, previous studies have also reported that lower SMI and sarcopenia are modifiable prognostic factors in patients with UBC who have undergone RC. For instance, Psutka et al. showed that preoperative sarcopenia was independently associated with both increased cancer-related death and all-cause mortality in a multivariable analysis (22). In another study by Hirasawa et al. involving patients with UBC, preoperative sarcopenia was a significant independent predictor of unfavorable outcome, clinical T stage, hydronephrosis, histological type of TUR-BT specimens, and neutrophil-to-lymphocyte ratio (12). Conversely, a report by Smith et al. implied that sarcopenia was not significantly associated with worse OS rate (23). In the present study, preoperative sarcopenia was not associated with any clinicopathological features or prognoses. However, sarcopenia one year after RC was significantly associated with various pathological features, including tumor state $(P=0.028)$, tumor grade $(P=0.029)$, and OS. We also observed that SMI change was a useful predictor of OS after RC. Therefore, we suggest that postoperative CT should be performed and that clinicians should check the SMI during follow-up in patients with UBC. Nutritional support and the prevention of cachexia might be needed in selected patients with UBC who have undergone RC.

Sarcopenia may also predict complications and OS among patients with advanced or metastatic UBC who have undergone RC $(21,23$, 24). In this regard, Wan et al. revealed that low SMI was frequently found in patients with BC who had undergone RC, and that this was strongly associated with early complications after surgery (24). Similarly, Smith et al. reported that sarcopenia was a predictor of major complications after $\mathrm{RC}$ in women, even after adjustment for known risk stratification characteristics (23). In advanced UBC, sarcopenia was useful in evaluating prognosis (21). More specifically, in a cohort of 88 patients with advanced UBC, the median OS rates were 11 and 31 months among sarcopenic and non-sarcopenic patients, respectively. In a multivariable analysis, sarcopenia was a significant and independent predictor of shorter OS (HR: 3.36). As sarcopenia reflects many clinical conditions, such as frailty, low nutritional status, active catabolism, and systemic inflammation, clinicians, including uro-oncologists, may use it for various purposes.

Several limitations of the current study must be acknowledged. First, the study had a retrospective design and involved a relatively small number of patients who underwent RC at a single institution. This may have led to sampling bias. Moreover, patients without available CT scans were excluded, which may also have caused selection bias. A prospective, randomized study involving a larger cohort and using multi-institutional methods will be required to confirm the present results. Second, the definition of sarcopenia was diverse in the present study. Although the volume of skeletal muscle mass differs according to ethnicity (25), the cut-off ranges defined in a previous Western study were within those determined in the present study. Considering ethnic and constitutional factors, a validated definition should be adopted in further studies. Despite these drawbacks, our present study presents a novel prognostic marker for predicting OS in patients with UBC who have undergone RC. The study indicated that the correction of sarcopenia after RC, as well as surveillance in selected patients, will improve postoperative management.

\section{CONCLUSIONS}

Sarcopenia and SMI changes one year after RC, which can be readily followed up using routine CT, might be effective predictors of OS in patients with UBC. This novel prognostic marker may assist in selecting patients with UBC who would benefit from nutritional support and interventions to prevent muscle wasting and consequent sarcopenia. The clinical utility of SMI changes as a prognostic marker merits further evaluation in prospective or external validation studies.

\section{ABBREVIATIONS}

$\mathrm{BC}=$ Bladder cancer

$\mathrm{RC}=$ Radical cystectomy

$\mathrm{MIBC}=$ muscle-invasive $\mathrm{BC}$

NMIBC $=$ non-muscle-invasive $B C$ 
SMI = skeletal muscle index

OS = overall survival

UBC $=$ urothelial bladder cancer

$\mathrm{CT}=$ computed tomography

TUR-BT = transurethral resection of bladder tumor

BCG = Bacille Calmette-Guerin

WHO = World Health Organization

$\mathrm{HU}=$ Hounsfield unit

$\mathrm{BMI}=$ body mass index

$\mathrm{HR}=$ hazard ratio

$\mathrm{CI}=$ confidence interval

\section{ACKNOWLEDGEMENTS}

The current research was supported by Kyungpook National University Research Fund, 2016.

\section{CONFLICT OF INTEREST}

None declared.

\section{REFERENCES}

1. Bak DJ, Lee YJ, Woo MJ, Chung JW, Ha YS, Kim HT, et al. Complications and oncologic outcomes following robotassisted radical cystectomy: What is the real benefit? Investig Clin Urol. 2016;57:260-7.

2. Ha YS, Yan C, Kim IY, Yun SJ, Moon SK, Kim WJ. Tissue hOGG1 genotype predicts bladder cancer prognosis: a novel approach using a peptide nucleic acid clamping method. Ann Surg Oncol. 2011;18:1775-81.

3. Ha YS, Kim TH: Chemotherapy in Advanced Urothelial Carcinoma. Korean. J Urol Oncol. 2016; 14: 47-53.

4. Ha YS, Kim MJ, Yoon HY, Kang HW, Kim YJ, Yun SJ, et al. mRNA Expression of S100A8 as a Prognostic Marker for Progression of Non-Muscle-Invasive Bladder Cancer. Korean J Urol. 2010;51:15-20.

5. Kim YH, Yan C, Lee IS, Piao XM, Byun YJ, Jeong P, et al. Value of urinary topoisomerase-IIA cell-free DNA for diagnosis of bladder cancer. Investig Clin Urol. 2016;57:106-12.

6. Aziz A, May M, Burger M, Palisaar RJ, Trinh QD, Fritsche HM, et al. Prediction of 90-day mortality after radical cystectomy for bladder cancer in a prospective European multicenter cohort. Eur Urol. 2014;66:156-63.
7. Svatek RS, Fisher MB, Matin SF, Kamat AM, Grossman $H B$, Nogueras-González GM, et al. Risk factor analysis in a contemporary cystectomy cohort using standardized reporting methodology and adverse event criteria. J Urol. 2010;183:929-34.

8. Makary MA, Segev DL, Pronovost PJ, Syin D, BandeenRoche $\mathrm{K}$, Patel $\mathrm{P}$, et al. Frailty as a predictor of surgical outcomes in older patients. J Am Coll Surg. 2010;210:901-8.

9. Osuna-Pozo CM, Serra-Rexach JA, Viña J, Gómez-Cabrera Mdel C, Salvá A, Ruiz D, et al. Prevalence of sarcopenia in geriatric outpatients and nursing homes. The ELLI study. Rev Esp Geriatr Gerontol. 2014;49:72-6.

10. Safer U, Gariballa S, Alessa A. Response to letter to the editor: Accurate diagnosis of sarcopenia in elderly requires correct measurement of muscle mass. Clin Nutr. 2013;32:663.

11. Tsukioka T, Nishiyama N, Izumi N, Mizuguchi S, Komatsu H, Okada S, et al. Sarcopenia is a novel poor prognostic factor in male patients with pathological Stage I non-small cell lung cancer. Jpn J Clin Oncol. 2017;47:363-8.

12. Hirasawa $Y$, Nakashima J, Yunaiyama D, Sugihara T, Gondo T, Nakagami Y, et al. Sarcopenia as a Novel Preoperative Prognostic Predictor for Survival in Patients with Bladder Cancer Undergoing Radical Cystectomy. Ann Surg Oncol. 2016;23(Suppl 5):1048-54.

13. Stein JP, Quek ML, Skinner DG. Lymphadenectomy for invasive bladder cancer. II. technical aspects and prognostic factors. BJU Int. 2006;97:232-7.

14. Osunkoya AO, Grignon DJ. Practical issues and pitfalls in staging tumors of the genitourinary tract. Semin Diagn Pathol. 2012;29:154-66.

15. Lopez-Beltran A, Bassi P, Pavone-Macaluso M, Montironi R. Handling and pathology reporting of specimens with carcinoma of the urinary bladder, ureter, and renal pelvis. Eur Urol. 2004;45:257-66.

16. Vrooman OP, Witjes JA. Follow-up of patients after curative bladder cancer treatment: guidelines vs. practice. Curr Opin Urol. 2010;20:437-42.

17. Mitsiopoulos N, Baumgartner RN, Heymsfield SB, Lyons W, Gallagher D, Ross R. Cadaver validation of skeletal muscle measurement by magnetic resonance imaging and computerized tomography. J Appl Physiol (1985). 1998;85:115-22.

18. Mourtzakis M, Prado CM, Lieffers JR, Reiman T, McCargar LJ, Baracos VE. A practical and precise approach to quantification of body composition in cancer patients using computed tomography images acquired during routine care. Appl Physiol Nutr Metab. 2008;33:997-1006. 
19. Prado CM, Lieffers JR, McCargar LJ, Reiman T, Sawyer $M B$, Martin L, et al. Prevalence and clinical implications of sarcopenic obesity in patients with solid tumours of the respiratory and gastrointestinal tracts: a populationbased study. Lancet Oncol. 2008;9:629-35.

20. Martin L, Birdsell L, Macdonald N, Reiman T, Clandinin MT, McCargar LJ, et al. Cancer cachexia in the age of obesity: skeletal muscle depletion is a powerful prognostic factor, independent of body mass index. J Clin Oncol. 2013;31:1539-47.

21. Fukushima H, Yokoyama M, Nakanishi Y, Tobisu K, Koga F. Sarcopenia as a prognostic biomarker of advanced urothelial carcinoma. PLoS One. 2015;10:e115895.

22. Psutka SP, Carrasco A, Schmit GD, Moynagh MR, Boorjian $S A$, Frank I, et al. Sarcopenia in patients with bladder cancer undergoing radical cystectomy: impact on cancer-specific and all-cause mortality. Cancer. 2014;120:2910-8.

23. Smith AB, Deal AM, Yu H, Boyd B, Matthews J, Wallen EM, et al. Sarcopenia as a predictor of complications and survival following radical cystectomy. J Urol. 2014;191:1714-20.
24. Wan F, Zhu Y, Gu C, Yao X, Shen Y, Dai B, et al. Lower skeletal muscle index and early complications in patients undergoing radical cystectomy for bladder cancer. World J Surg Oncol. 2014:12:14

25. Wulan SN, Westerterp KR, Plasqui G. Ethnic differences in body composition and the associated metabolic profile: a comparative study between Asians and Caucasians. Maturitas. 2010;65:315-9.

\section{Correspondence address}

Eun Sang Yoo, MD

Department of Urology,

Kyungpook National University Hospital

School of Medicine, Kyungpook National University

680 Gukchaebosang-ro

Jung-gu, Daegu 41944, Republic of Korea

Fax: +82 534 21-9618

E-mail: uroyoo@knu.ac.kr 